\title{
用于提升金属-氮-碳催化剂氧还原活性的双金属自旋态 调控策略
}

于吉红

吉林大学化学学院, 无机合成与制备化学国家重点实验室, 长春 130012

E-mail: jihong@jlu.edu.cn

\section{Regulating Fe-spin state by atomically dispersed Mn-N in Fe-N-C catalysts with high oxygen reduction activity}

\section{Jihong $\mathrm{Yu}$}

State Key Laboratory of Inorganic Synthesis and Preparative Chemistry, College of Chemistry, Jilin University, Changchun 130012, China E-mail: jihong@jlu.edu.cn

doi: 10.1360/TB-2021-0145

因其高的电导率和独特的金属-配体相互作用, 过渡金 属-氮-碳(M-N-C)催化剂具有优异的氧气还原(ORR)催化性 能, 在燃料电池、金属空气电池等能源电催化领域展现出很 大的应用潜力 ${ }^{[1]}$, 被认为是替代商业 $\mathrm{Pt} / \mathrm{C}$ 催化剂的极具潜力 的新型催化剂. 因此, 如何构建高性能的M-N-C催化剂, 使其 性能接近或超越 $\mathrm{Pt} / \mathrm{C}$ 催化剂, 成为其替代贵金属催化剂的关 键. 这对于燃料电池和金属空气电池的产业化应用和可持续 发展具有重要意义.

理论研究表明, 过渡金属 $(\mathrm{M}=\mathrm{Mn}, \mathrm{Fe}, \mathrm{Co}, \mathrm{Ni}$ 等 $)$ 的3d未 占据轨道可以容纳外来电子, 从而降低 $\mathrm{OOH}^{*}, \mathrm{O}^{*} / \mathrm{OH}^{*}$ 等中 间体的键合强度, 从而具有催化还原 $\mathrm{O}_{2}$ 的潜力 ${ }^{[2]}$. 催化剂的活 性主要受其电子结构的影响, 可以通过打破催化剂表面的 $\mathrm{M}^{(m+1)+}-\mathrm{O}_{2}^{2-}$ 键来释放能量, 以确保 $\mathrm{O}^{2-} / \mathrm{OH}$ 的快速转换和 $\mathrm{OH}$ 的再生 ${ }^{[3]}$. 基于配位环境, $\mathrm{Fe}^{\mathrm{III}}$ 具有多种自旋状态, 包括低自旋 $\mathrm{t}_{2 \mathrm{~g}} 5 \mathrm{e}_{\mathrm{g}} 0$, 中自旋 $\mathrm{t}_{2 \mathrm{~g}} 4 \mathrm{e}_{\mathrm{g}} 1$ 和高自旋 $\mathrm{t}_{2 \mathrm{~g}} 3 \mathrm{e}_{\mathrm{g}} 2$. 低自旋电子构型为 $\mathrm{d}_{x y} 2 \mathrm{~d}_{y z} 2 \mathrm{~d}_{x z}$, 没有电子占据反键轨道, 从而导致 $\mathrm{M}^{m+} / \mathrm{O}_{2}$ 强的 相互作用和稳定的 $\mathrm{M}^{(m+1)+}-\mathrm{O}_{2}{ }^{2-}$ 键, 使 $\mathrm{M}^{(m+1)+}-\mathrm{O}_{2}{ }^{2-} / \mathrm{M}^{m+}-\mathrm{OOH}$ 跃迁变得困难 ${ }^{[4]}$; 高自旋电子的构形是 $\mathrm{d}_{x y} 1 \quad \mathrm{~d}_{y z} 1 \mathrm{~d}_{x z} 1 \mathrm{~d}_{z}{ }^{2} 1$ $\mathrm{d}_{x}{ }^{2}-y{ }^{2} 1$, 其高的填充度 $\left(\mathrm{d}_{z}{ }^{2} 1 \mathrm{~d}_{x}{ }^{2}-{ }^{2} 1\right)$ 导致吸附能力差和性能 差 ${ }^{[5]}$; 而对于中自旋电子构型 $\left(\mathrm{d}_{x y} 2 \mathrm{~d}_{y z} 1 \mathrm{~d}_{x z} 1 \mathrm{~d}_{z}^{2} 1\right)$, 中等自旋态 的单个 $\mathrm{d}_{z}{ }^{2}$ 电子可以轻松穿透氧的反键 $\pi$ 轨道, 从而具有很高 的ORR活性. 因此, 通过改善过渡金属的周边化学环境, 调制 其电子自旋状态可以精确调控催化位点的电子结构, 有望获 得活性可调的高性能ORR催化剂.

郑州大学张佳楠课题组及其合作者 ${ }^{[6]}$ 提出提升金属-氮-
碳催化剂ORR活性的双金属自旋态调控新策略(图1). 他们采 用预聚合和热解方法制备了 $\mathrm{Fe}, \mathrm{Mn}$ 双金属原子分散的 $\mathrm{Fe}$, $\mathrm{Mn} / \mathrm{N}-\mathrm{C}$ 电催化剂. 磁性测量表明, Mn-N结构的引人导致 $\mathrm{Fe}^{\mathrm{III}}$ 电子离域, 并使 $\mathrm{Fe}^{\mathrm{III}}$ 的自旋态从低自旋 $\left(\mathrm{t}_{2 \mathrm{~g}} 5 \mathrm{e}_{\mathrm{g}} 0\right)$ 转变为中自旋 $\left(\mathrm{t}_{2 \mathrm{~g}} 4 \mathrm{e}_{\mathrm{g}} 1\right)$, 从而易于穿透氧的反键 $\pi$ 轨道. 密度泛函理论(DFT) 计算表明, 自旋态精确可调的 $\mathrm{Fe}, \mathrm{Mn} / \mathrm{N}-\mathrm{C}$ 可以适度地与氧相 互作用，具有优化的键长和吸附能，可以促进ORR反应的动

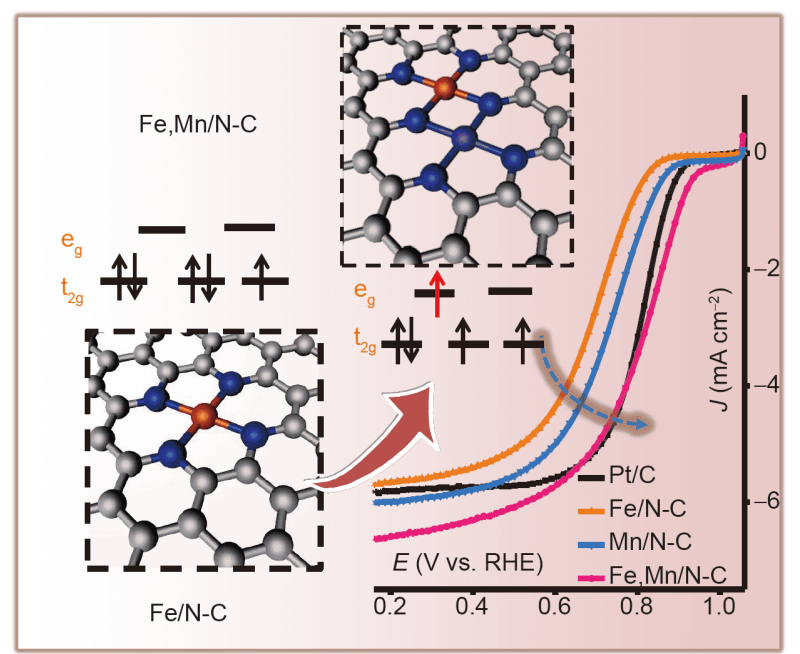

图 1 (网络版彩色) 原子级 $\mathrm{Mn}-\mathrm{N}$ 掺杂调控 $\mathrm{Fe}-\mathrm{N}_{x}$ 自旋态提升酸性 ORR性能

Figure 1 (Color online) Regulating $\mathrm{Fe}-\mathrm{N}_{x}$ spin state by atomically dispersed $\mathrm{Mn}-\mathrm{N}$ with high oxygen reduction activity in acid medium 
力学过程. 实验表明, 该催化剂在碱性和酸性介质中均表现出 优异的ORR性能(在 $0.1 \mathrm{~mol} / \mathrm{L} \mathrm{KOH}$ 中半波电位为 $0.928 \mathrm{~V}$, 在 $0.1 \mathrm{~mol} / \mathrm{L} \mathrm{HClO}_{4}$ 中半波电位为 $\left.0.804 \mathrm{~V}\right)$; 具有良好耐久性, 在 碱性和酸性介质中, 分别优于和近似于商用 $\mathrm{P} t / \mathrm{C}$ 的活性. 此外, 该催化剂在可逆锌空气电池中也具有优异的功率密度特性和 长期耐用性. 总之, 基于原子分散的双金属结构, 可以精确调 控活性金属的自旋态，从而获得高活性的Fe-N-C催化剂. 该成 果为金属-氮-碳活性位点的调控开创了新的路径, 对高效低成
本非贵金属催化剂的研究和开发具有重要的指导价值.

上述工作2021年3月在线发表于Nature Communications. 近年来，张佳楠团队致力于研发杂原子掺杂的缺陷碳金属复 合催化材料, 通过异原子的引人调控催化活性中心电子结构, 使其活性和稳定性得到显著提高, 相关研究成果在Advanced Materials, Advanced Functional Materials, ACS Nano 等期刊 上发表 ${ }^{[7-9]}$. 该系列工作为构建具有优异ORR性能的金属-氮碳催化剂提供了便捷高效的策略和路径.

\section{参考文献}

1 Zhao L, Zhang Y, Huang L B, et al. Cascade anchoring strategy for general mass production of high-loading single-atomic metal-nitrogen catalysts. Nat Commun, 2019, 10: 1278

2 Wang H, Liu R, Li Y, et al. Durable and efficient hollow porous oxide spinel microspheres for oxygen reduction. Chem, 2018, 2: 337-348

$3 \mathrm{Mu}$ C, Mao J, Guo J, et al. Rational design of spinel cobalt vanadate oxide $\mathrm{Co}_{2} \mathrm{VO}_{4}$ for superior electrocatalysis. Adv Mater, 2020, 32 : 1907168

4 Wang X T, Ouyang T, Wang L, et al. Redox-inert $\mathrm{Fe}^{3+}$ ions in octahedral sites of Co-Fe spinel oxides with enhanced oxygen catalytic activity for rechargeable zinc-air batteries. Angew Chem Int Ed, 2019, 58: 13291-13296

5 Shen G, Zhang R, Pan L, et al. Regulating the spin state of $\mathrm{Fe}^{\mathrm{III}}$ by atomically anchoring on ultrathin titanium dioxide for efficient oxygen evolution electrocatalysis. Angew Chem Int Ed, 2020, 59: 2313-2317

6 Yang G, Zhu J, Yuan P, et al. Regulating Fe-spin state by atomically dispersed Mn-N in Fe-N-C catalysts with high oxygen reduction activity. Nat Commun, 2021, 12: 1734

7 Qiao Y, Yuan $\mathrm{P}, \mathrm{Hu} \mathrm{Y}$, et al. Sulfuration of an $\mathrm{Fe}-\mathrm{N}-\mathrm{C}$ catalyst containing $\mathrm{Fe}_{x} \mathrm{C} / \mathrm{Fe}$ species to enhance the catalysis of oxygen reduction in acidic media and for use in flexible Zn-air batteries. Adv Mater, 2018, 30: e1804504

8 Guo Y, Yuan P, Zhang J, et al. $\mathrm{Co}_{2}$ P-CoN double active centers confined in N-doped carbon nanotube: Heterostructural engineering for trifunctional catalysis toward HER, ORR, OER, and Zn-air batteries driven water splitting. Adv Funct Mater, 2018, 28: 1805641

9 Guo Y, Yuan P, Zhang J, et al. Carbon nanosheets containing discrete Co- $\mathrm{N}_{x}-\mathrm{B}_{y}$-C active sites for efficient oxygen electrocatalysis and rechargeable Zn-air batteries. ACS Nano, 2018, 12: 1894-1901 